\title{
Precise Multi-Spectral Dermatological Imaging
}

\author{
David Delgado Gómez, Jens Michael Carstensen, Bjarne Kjær Ersbøll \\ Informatics and Mathematical Modelling, Section of Image Analysis and Computer Graphics \\ Technical University of Denmark
}

\begin{abstract}
In this work, an integrated imaging system to obtain accurate and reproducible multi-spectral dermatological images is proposed. The system is made up of an integrating sphere, light emitting diodes and a generic monochromatic camera. The system can collect up to 10 different spectral bands. These spectral bands vary from ultraviolet to near infrared. The welldefined and diffuse illumination of the optically closed scene aims to avoid shadows and specular reflections. Furthermore, the system has been developed to guarantee the reproducibility of the collected images. This allows for comparative studies of time series of images.

Two experiments are conducted to show the ability of the system to acquire highly precise and standardized multi-spectral images. The first experiment aims to show the capacity of the system to collect reproducible images. The second experiment demonstrates that the multi-spectral images provide more information than the classical thri-chromatic images and that this information is enough to segment lesions easily. These two facts together indicate the suitability of the system to collect images and to summarize and track the evolution of dermatological diseases.
\end{abstract}

Index Terms-Image acquisition, calibration, illumination, independent component analysis, projection pursuit.

\section{INTRODUCTION}

Nowadays, dermatological diseases are among the main reasons that makes people visit physicians. E.g., psoriasis affects between the 1 and 2 percent of the population of the United States and United Kingdom. More than 3 million people receive treatment every year in these two countries only for psoriasis. A considerable amount of money is invested in treatments and consultation. In the United States, the total annual expenditure for cutaneous psoriasis exceeded $\$ 1.5$ billion without taking into account the use of cyclosporine (currently the most expensive systematic treatment available) [1]. This amount of money could make one think that the evolution of the lesion is controlled with precise and sophisticated high tech equipment. However, currently, the diagnosis of skin lesions is imprecise. There are no objective methods to evaluate the lesion. Physicians visually assess the lesion and make scorings and journal notes of the condition. These notes and perhaps some photographs are presently the only memory of what the lesion looked like at the corresponding visit. Consequences of this doctor dependence are inaccurate evaluation of new treatments and the non-reliability of the case history when a patient has to be treated by another doctor.

Many research projects has been conducted in the last years to give an alternative to these rudimentary methods. In general, these projects can be divided in two major categories. The first category is composed of projects that aim to develop an accurate system to collect suitable dermatological images.
These works have focused on developing precise calibrations that guarantee the quality of the images [2] [3]. However, in many research projects, the reproducibility of the images is not considered [4]. This means that a preprocessing is required before images collected at different times can be compared[5].

The second group is made up of work that aims to characterize and quantify the severity of the lesion. E.g., Kjolen [6] demonstrated that features extracted from color skin tumor images by computer vision methods can be reliable discriminators of malignant tumors from benign ones. In a more recent work, Hillebrand [7] used computer analysis in high resolution digital images to compare the skin condition of a group of females. Although image analysis methods have provided good alternatives to many dermatological problems, sometimes, the provided solution is complex or it requires a preprocessing of the images. This is due to that, in some occasions, the information provided by tri-chromatic images is insufficient. A few non-imaging projects have appeared considering the inclusion of extra bands (mainly near infrared bands) to help to characterized the lesion [8]. However, most of the actual works are still applied on RGB images (or transform of this image to a more suitable three dimensional color space as HSV or YCBCR)[9].

In this work, a novel image system is proposed to acquire dermatological images. The system captures up to 10 different bands (ranging from ultra-blue to near infrared) in order to provide enough information to characterize the lesion. Moreover, the system has been developed with the goal of obtaining standardized images so that images collected at different times can be compared without any preprocessing.

\section{DESCRIPTION OF THE EQUIPMENT}

The proposed equipment, developed by Videometer ${ }^{1}$, is composed of a camera, light emitting diodes and a integrating sphere. The equipment has been designed to produce completely diffuse light to avoid shadows and specular reflections, and to acquire multi-spectral images by fast strobe illumination from light emitting diodes(LEDs) at up to ten different wavelengths.

Figure 1 shows the equipment. Figure 1 right displays a sketch of the set-up. It displays the position of the camera and the diodes inside of the sphere and the place where the lesion is located. Figure 2 left displays the position of the diodes inside the equipment.

The camera resolution is a $1380 \times 1035$. The nine different wavelengths used in this study are: $472 \mathrm{~nm}, 503 \mathrm{~nm}, 515 \mathrm{~nm}$,

\footnotetext{
${ }^{1}$ www.videometer.com
} 

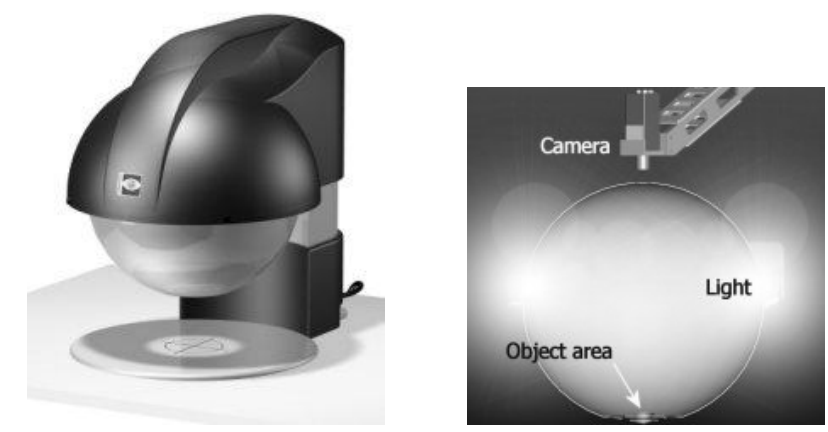

Fig. 1. Left: image of the system. Right: position of the camera and the light sources with respect to the object area.
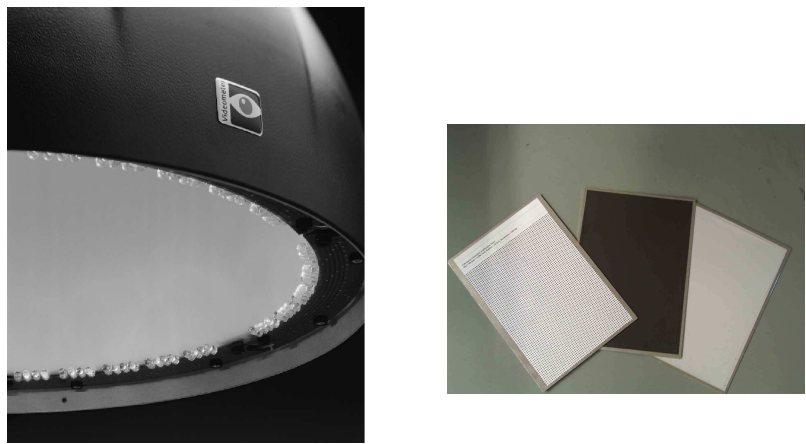

Fig. 2. Left: location of the diodes inside of the sphere. Right: calibration sheets.

$592 \mathrm{~nm}, 612 \mathrm{~nm}, 630 \mathrm{~nm}, 700 \mathrm{~nm}, 875 \mathrm{~nm}(\mathrm{NIR})$, and 940 $\mathrm{nm}(\mathrm{NIR})$.

In order to increase the accuracy and reproducibility of the images a radiometric and a geometric calibration are conducted [10]. The radiometric calibration aims to eliminate problems with uneven intensities and vigneting, and to standardize the measurement scale. With this goal in mind two sheets of the natural color system (NCS) from Scandinavian Color Institute were selected as calibration targets (NCS 1500 and NCS 8000). The equipment collects an image of each sheet. Then a non-linear calibration function is estimated and applied to each image pixel during the further image acquisition. The geometric calibration is conducted to make sure that aberrations, such as distortion, decentering and thin prism aberration, do not affect the accuracy of the images. An image of a white sheet with black spots is grabbed with the camera for each wavelength. This calibration target is shown in figure 2 right, together with the radiometric sheets. The collected multi-spectral images are thresholded and the center of gravity of each spot is calculated. A third order polynomial is applied to warp the centers of gravity to a given target. This is done for each band in the multi-spectral image in order to assure co-site registration.

\section{Evaluation of THE IMAGES}

The analysis of RGB dermatological images has proved to be difficult when hair or vessels are present in the images [11] [12]. In order to check if the inclusion of the extra bands helps to solve this problem, a projection pursuit [13] in 1 and 2 dimensions is conducted. Friedman [14] developed the projection pursuit as a tool to find interesting structures in the data. Interesting structures are found via linear projections in which the distribution of the projected data differs as much as possible from the Gaussian distribution. Friedman justifies the non-interest of the normal distribution based on a series of properties as all the projections of a multivariate normal distribution are normal or that, for a fixed variance, the normal distribution has the least information (Fisher, negative entropy). The deviation from a Gaussian is measured through an index that measures the non-normality of the projected data. In $1 \mathrm{D}$, Friedman looks for a projection of the sphered data $\mathrm{Z}$, $X=\alpha^{T} Z$, such that the index

$$
I(\alpha)=\frac{1}{2} \sum_{j=1}^{J}(2 j+1)\left[\frac{1}{N} \sum_{i=1}^{n} P_{j}\left(2 \Phi\left(\alpha^{T} z_{i}\right)-1\right)\right]^{2}
$$

is maximized. $P_{j}$ is the Legendre polynomium of order $\mathrm{j}$ and $\Phi(X)$ is the standard normal density.

Friedman proposed an extension to find 2D projections. However, in this work the index developed by Posse [17] [18] is preferred due to the computational properties.

Once an interesting projection has been found, the structure that makes the projection interesting is removed and the algorithm looks for a next informative view. This process consists in transforming the data so that the density of the transformed data $Z^{k+1}$ is as close as possible to the old data $Z^{k}$ under the constraint that its marginal density is normal. This produces the closest distribution in the sense of relative entropy distance measure

$$
\int \log \left(Z^{K} / Z^{k+1}\right) Z^{k} d Z
$$

In this work, the structure removal proposed by Friedman is used both for $1 \mathrm{D}$ and $2 \mathrm{D}$. In 1D, the transformed data are given by

$$
Z^{k+1}=U^{T} \Theta\left(U Z^{k}\right)
$$

where $\mathrm{U}$ is an orthonormal $(q \times q)$ matrix with $\alpha$ as the first row and $\Theta$ a vector transformation such us $\Theta(T)$ is given by

$$
\Theta\left(T_{1}\right)=\Phi^{-1}\left(F_{\alpha}(T 1)\right)
$$

$\Theta\left(T_{j}\right)=T_{j}$, where $j>1$ is the row number

being $F_{\alpha}(T 1)$ the cumulative distribution function. The extension to $2 \mathrm{D}$ is based in the same principle.

\section{EXPERIMENTAL RESULTS}

Two experiments are conducted to test the suitability of the equipment to evaluate the severity of dermatological lesions and to detect and measure changes during their treatment.

\section{EXPERIMENT 1: REPRODUCIBILITY OF MEASUREMENTS}

The objective of the first experiment is to evaluate the precision of the equipment. Precision is, in this case, defined as the reproducibility of measurements, or the way that repeated measurements are spread around the average of those measurements [15]. In dermatology, this quality is of prime 


\begin{tabular}{|c|c|c|c|c|}
\hline Band/NCS number & $1500 \mathrm{~N}$ & $2500 \mathrm{~N}$ & $5000 \mathrm{~N}$ & $8500 \mathrm{~N}$ \\
\hline Blue 472 & 0.0007 & 0.0105 & 0.0236 & 0.0348 \\
\hline Green 515 & 0.0002 & 0.0012 & 0.0028 & 0.0074 \\
\hline Amber 592 & 0.0013 & 0.0371 & 0.1295 & 0.1563 \\
\hline Red 630 & 0.0012 & 0.0078 & 0.0199 & 0.0222 \\
\hline Near IR 875 & 0.0010 & 0.0062 & 0.0434 & 0.0366 \\
\hline Ultra Blue 428 & 0.0058 & 0.0057 & 0.0141 & 0.0320 \\
\hline Cyan 503 & 0.0003 & 0.0011 & 0.0023 & 0.0086 \\
\hline Orange 612 & 0.0004 & 0.0066 & 0.0234 & 0.0319 \\
\hline Near IR 940 & 0.0001 & 0.0076 & 0.0501 & 0.0726 \\
\hline
\end{tabular}

TABLE I

VARIANCE OF THE SEVEN MEANS OBTAINED FOR EACH NCS SHEET IN EACH SPECTRAL BAND

importance. It ensures that differences in measurements of the lesion obtained at different times are consequence of the evolution of the disease, and not to different ambient conditions.

In order to assess the reproducibility of the images, the equipment was kept turned on during 7 hours. The set-up was calibrated every hour and images of four Natural Color System sheets( $1500 \mathrm{~N}, 2500 \mathrm{~N}, 5000 \mathrm{~N}, 8500 \mathrm{~N})$ from Scandinavian Color Institute were collected. The NCS sheets are all painted and have very small variation.

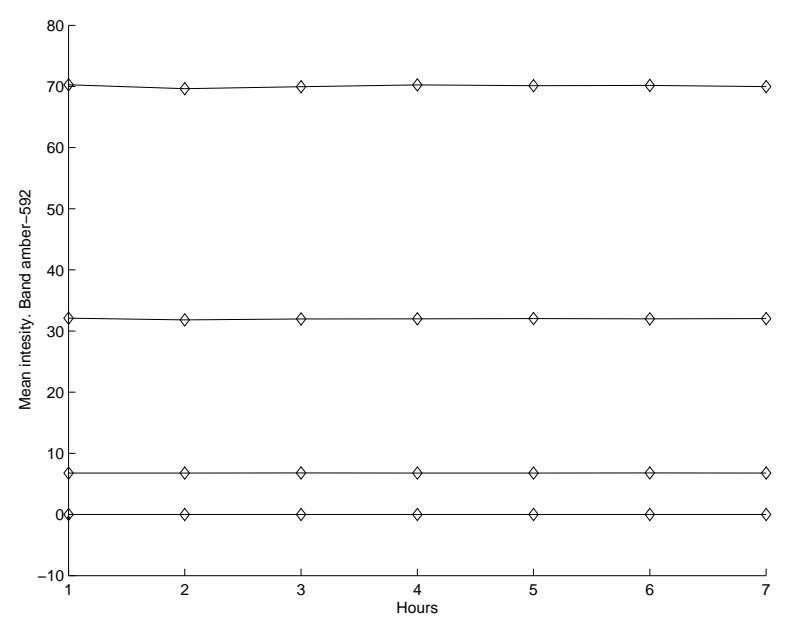

Fig. 3. Variation in the measurements of the NCS respect to the time that the equipment was turned on in the amber band, $592 \mathrm{~nm}$.

The mean of each spectral bands of the collected images was calculated. If the system performs accurately, the mean should not vary significant with respect to time. Marks were placed in the NCS sheets to calculate the mean in approximately the same area.

Figure 3 shows the evolution of the measures with respect to time of the four NCS sheets in the amber band (592 $\mathrm{nm}$ ). Results obtained in the other bands are similar to the obtained in this band. From the figure, it is noticed that the variation is minimal. After the first hour, where the equipment reached thermal equilibrium, the differences are unappreciable.

Moreover, for fixed NCS sheet, the variance of the obtained measurements for each band is minimal. In table I, the variance of the measurements obtained for each band of the different NCS sheets is displayed. This small variance guarantees that measures of the dermatological lesions, obtained at different times, depend only on the changes in the lesion. Therefore, the system is appropriate from the point of view of robustness.

\section{EXPERIMENT 2: ASSESSMENT OF THE INFORMATION PROVIDED BY THE MULTI-SPECTRAL IMAGES IN TERMS OF SEGMENTATION AND LESION CHARACTERIZATION.}

The second experiment aims to statistically assess the information provided by the six extra bands to segment and characterize the lesion. In order to accomplish this goal, nine multi-spectral images were collected from four different psoriasis patients. These images were obtained in collaboration with the dermatological department of Gentofte Hospital in Denmark. The nine different psoriasis images are displayed in figure 4 (A). The nine different spectral bands of the top left image with their corresponding wavelengths are displayed in figure 4(B).

From these raw images, it can be noticed that the nearinfrared bands, (the last two images in figure 4(B)), are able to localize the veins. The near infrared band $940 \mathrm{~nm}$ is displayed for each image in figure 4 (C). These facts suggest that the characterization of the lesion including the near-infrared bands will be more precise.

A one dimensional projection pursuit is conducted with the objective of comparing the information provided by the six extra bands in order to find structures in the image. Five projections are found for each image using the nine spectral bands and five other projections are calculated using only the RGB bands.

Figure 4 (D) displays the projections obtained in one of the images. Results for the other images are similar to the obtained with this image. Looking at this figure, it is noticed, not surprisingly, that when only the RGB bands are used, the algorithm has difficulties in finding more than three structured projections. On the other hand, when all the bands are utilized, the algorithm can find at least five informative views where the lesion, hairs, scales or body contour are characterized. The extra bands are able to characterize more structures involved in the images than just the RGB bands. This could be used to remove the hair or occlusions that might appear in the image and to obtain more precise evaluations of the lesions. Moreover, it is noticed that a projection is found where the lesion exhibits a considerable contrast with respect to the other structures involved in the image. The projection where the lesion is enhanced for each image is shown in figure 5 (A).

The lesion can easily be segmented using these projections. The data in these projections are distributed approximately according to a mixture of two Gaussians. The parameters of this model can be estimated [19] and the lesion extracted via discriminant analysis. Results of the segmentation are shown in figure $5(\mathrm{~B})$. It is observed that the nine multi-spectral bands provide enough information to precisely separate the lesion from the other parts of the images. A projection pursuit in two dimensions was also conducted. However, results were not significantly different to the ones obtained with projection pursuit in terms of segmentation. Therefore, projection pursuit in one dimension is preferred because of the computation time. 


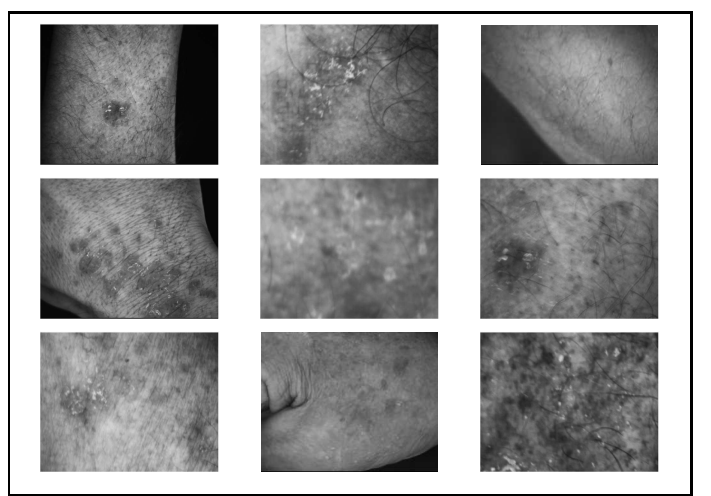

(A)

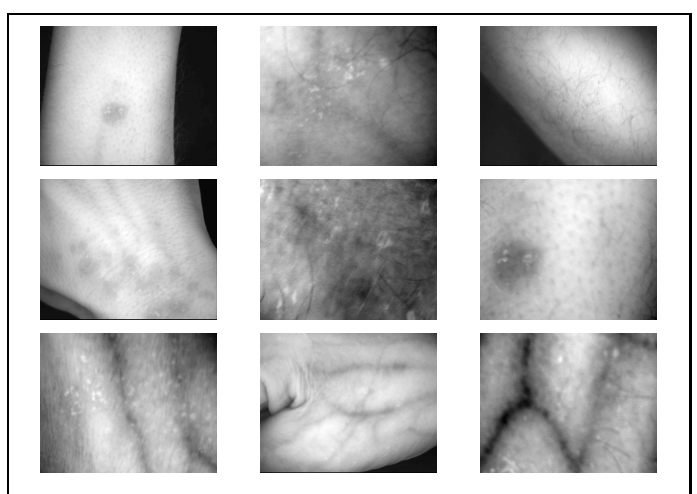

(C)

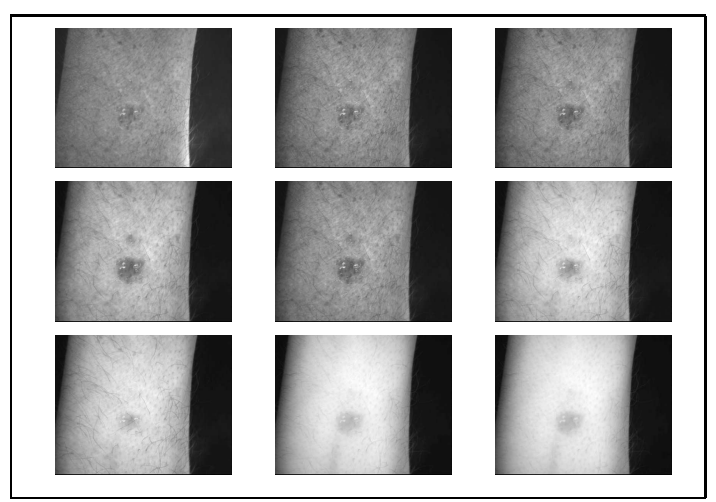

(B)

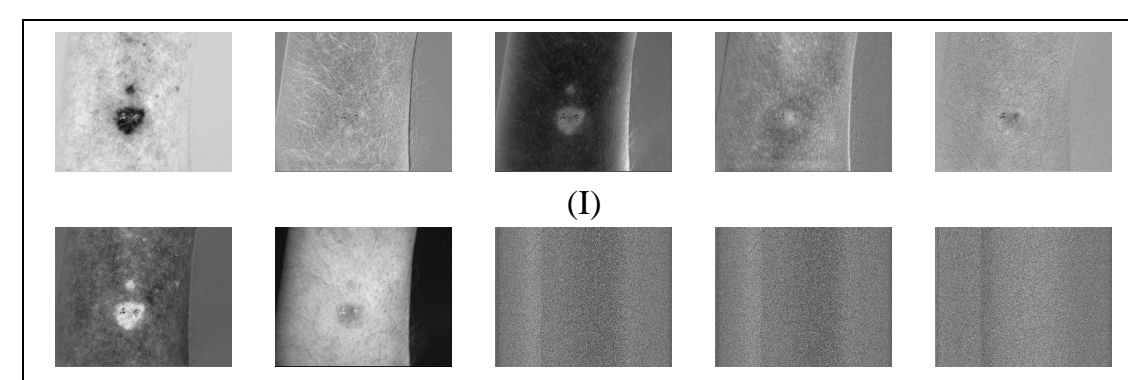

(II)

(D)

Fig. 4. (A): nine psoriasis images. (B): the nine multi-spectral bands of one of the images. Top Left: ultra-blue, 428. Top Center: blue, 472. Top Right: Cyan, 503. Middle Left: green, 515. Middle Center: amber, 592. Middle Right: orange, 612. Bottom Left: red, 630. Bottom Center: near infrared 875. Bottom Right: near infrared 940.(C): near infrared spectral band of each collected image. (D): Projections obtained using Friedman's projection pursuit on a psoriasis image. Top row (I) using the nine spectral bands, and bottom row (II) using just the RGB bands.
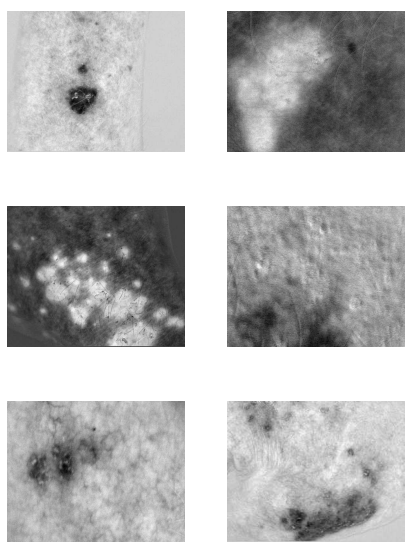

(A)
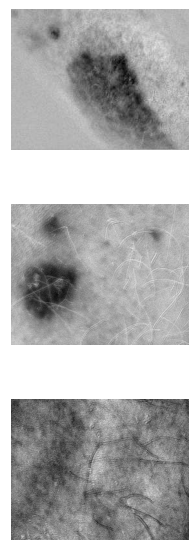
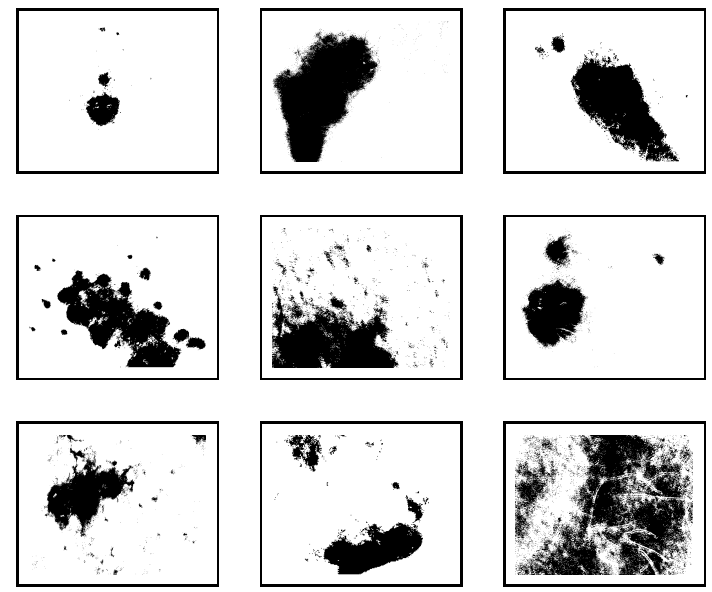

(B)

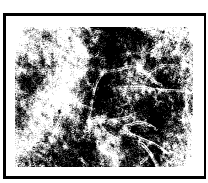

Fig. 5. (A): Obtained projections where the lesion is enhanced. (B): Segmentation of the different lesions.

The segmented images can be used to assess the information provided by the extra bands in terms of Mahalanobis distances between classes. Given two classes $\mathrm{X}$ and $\mathrm{Y}$ with observations $X_{1}, \ldots, X_{n_{1}}$ belonging to $\mathrm{X}$ and observations $Y_{1}, \ldots, Y_{n_{2}}$ belonging to $\mathrm{Y}$, Mahalanobis distance between $\mathrm{X}$ and $\mathrm{Y}$ is defined by

$$
\left(\mu_{1}-\mu_{2}\right)^{t} \Sigma^{-1}\left(\mu_{1}-\mu_{2}\right)
$$

where $\mu_{1}$ and $\mu_{2}$ are the mean of classes $\mathrm{X}$ and $\mathrm{Y}$ respectively and $\Sigma$ is defined by

$\Sigma=\frac{1}{n_{1}+n_{2}-2}\left(\sum_{i}\left(X_{i}-\bar{X}\right)\left(X_{i}-\bar{X}\right)^{t}+\sum_{i}\left(Y_{i}-\bar{Y}\right)\left(Y_{i}-\bar{Y}\right)^{t}\right.$

The mahalanobis distances, for each of the nine images, between the lesion and the class composed of the other structures in the image(healthy skin, hair,...) are shown in table II. It can be observed that the distance increase considerably. However, a more meaningful measure based on these 


\begin{tabular}{|c|c|c|}
\hline Image & $\begin{array}{c}\text { Mahalanobis } \\
\text { distance using } \\
\text { the RGB bands }\end{array}$ & $\begin{array}{c}\text { Mahalanobis } \\
\text { distance using } \\
\text { the nine bands }\end{array}$ \\
\hline top-left & 1.4142 & 2.0737 \\
\hline top-center & 1.1820 & 3.5039 \\
\hline top-right & 0.1344 & 0.4091 \\
\hline middle-left & 1.1164 & 1.4260 \\
\hline middle-center & 0.3346 & 0.8514 \\
\hline middle-right & 1.7280 & 2.7719 \\
\hline bottom-left & 1.0924 & 1.6107 \\
\hline bottom-center & 0.3446 & 0.4188 \\
\hline bottom-right & 0.2379 & 0.5726 \\
\hline
\end{tabular}

TABLE II

MAHALANOBIS DISTANCES BETWEEN THE LESION AND THE OTHER STRUCTURES INVOLVED IN THE IMAGE.

measures is to statistically test the null hypothesis that the six extra bands does not contribute to a better discrimination. Specifically, if the extra six variables do not contribute to a better discrimination, then

$$
Z=\frac{n_{1}+n_{2}-p-1}{q} \frac{n_{1} n_{2}\left(D_{p}-D_{q}\right)}{\left(n_{1}+n_{2}\right)\left(n_{1}+n_{2}-2+n_{1} n_{2} D_{q}\right)}
$$

follows a $F\left(q, n_{1}+n_{2}-p-1\right)$ distribution, where $n_{1}$ and $n_{2}$ are the number of observations of each class, $\mathrm{p}$ is the total number of variables, $q$ is the number of variables that are to be tested if they or not contribute to a better discrimination and $D_{p}$ and $D_{q}$ are the mahalanobis distances between classes using all the variables and all the variables except the last $q$. Results showed that statistically the null hypothesis was rejected with a significance level of 0.01 . This means that the last six variables strongly contribute to a better discrimination.

In order to assess which of the bands contribute more to a better discrimination, a forward selection was conducted. Initially, a set composed of the RGB bands and one of the extra spectral bands was created. The previous test was conducted for each extra band in order to obtain the test statistic Z. It was observed that the bands where the null hypothesis was most strongly rejected are the near infrared and the cyan. The spectral band where the null hypothesis is most strongly rejected was included in the RGB set and the process iterated. The final set, obtained including variables that are significant with a level of 0.01 , was composed of all the bands except the near infrared 940 and the orange 612. Therefore, the inclusion of four bands contribute to a better discrimination of the lesion.

\section{CONCLUSION}

In this work, a system to collect precise and reproducible multi-spectral dermatological images has been proposed. The system can collect up to nine different spectral bands. These bands are composed by the RGB tri-chromatic spectral bands plus six extra bands that can be choosen in the range going from ultra-blue to near infrared(from $400 \mathrm{~nm}$ to $1000 \mathrm{~nm}$ ). The reproducibility of the equipment has been tested. Results indicate that the equipment is a suitable tool to measure changes in the evolution of the disease. Furthermore, it has been observed that the six extra bands provide more information than the classical RGB images. This information can be used to remove noise such as hair or occlusions and to obtain more precise measures to characterize the lesion.

\section{ACKNOWLEDGMENT}

The authors would like to thank to the SITE Project funded by a grant from the Danish Technical Research Foundation (Project Number STVF 56-00-0123) for supporting the present work. The author would also like to thanks to the dermatologists Lone Skov and Bo Bang of Gentofte Hospital of Denmark and to the anonymous patients, for their collaboration during the image acquisition sessions.

\section{REFERENCES}

[1] C. Camisa, "Handbook of psoriasis",Blackwell Science, 1998.

[2] E. Marszalec, M. Pietikanen, "Online Color Camera Calibration", Proceedings of the 12th IAPR International Conference in Pattern Recognition, vol 1.

[3] A. Gutenev, V. N. Skladnev, D. Varvel, "Acquisition-time image quality in digital dermatoscopy of skin lesions", Computerized Medical Imaging and graphics, vol. 25, pp. 495-499, 2001.

[4] I. Maglogianis, "Automated Segmentation and Registration of Dermatological Images", Journal of Mathematical Modelling and Algorithms, Vol 2. Issue 3, pp. 277-294, 2003

[5] I. Ikeda, K. Urushihara, T. Ono, "A pitfall in clinical photography: the appearance of skin lesions depends upon the illumination device", Arch. Dermatol. Res, vol 294, pp. 438-443, 2003.

[6] A. Kjolen, M. J. Thompson, S.E. Umbaugh, R. H. Moss, W.V. Stoecker, "Performance of AI methods in detecting melanoma", IEEE Engineering in Medicine and Biology Magazine , Vol. 14, No 4, pp. 411-416, 1995.

[7] G. Hillebrand G., K. Miyamoto, B. Schnell, M. Ichihash, R. Shinkura, S. Akiba, "Quantitative evaluation of skin condition in an epidemiological survey of females living in northern versus southern Japan", Journal of Dermatological Science, vol. 27, pp. 42-52, 2001.

[8] L. M. McIntosh, M. Jackson, H. H. Mantsch, J. R. Mansfield, A. Crowson, J. Toole, "Near-infrared spectroscopy for dermatological applications", Vibrational Spectroscopy 28, pp. 53-58, 2002.

[9] M. Nischik, C. Forster, "Analysis of skin Erythema using true-color images",IEEE Transactions on Medical Imaging, Vol. 16, No. 6, December 1997.

[10] J. Folm-Hansen, "On Chromatic and Geometrical Calibration",PhD tesis, Lyngby, 1999.

[11] P. Schmid-Saugeon, J. Guillod, J. P. Thiran, "Towards a computeraided diagnosis system for pigmented skin lesions"Computerized Medical Imaging and Graphics, Vol. 27, pp. 65-78, 2003.

[12] T. Lee, V. Ng, R. Gallager, A. Coldman, D. McLean. "DullRazor: a software approach to hair removal from images". Compyt. Biol. Med., Vol. 27, pp. 629-639, 1997.

[13] M. C. Jones, R. Sibson, "What is Projection Pursuit", J. R. Statisti. Soc., Vol. 10, No 6, pp. 989-1003, 1987.

[14] J. Friedman, "Exploratory Projection Pursuit", Journal of the american Statistical Association, Vol. 82, No 397, pp., 249-269, 1987

[15] Y. Vander Haeghen, J. M. Naeyaert, I. Lemahieu, "An imaging system with calibrated color image acquisition for use in dermatology", IEEE transactions on medical imaging, Vol. 19, No. 7, july 2000.

[16] I. Maglogianis, "Design and Implementation of a Calibrated Store and Forward Imaging System for Teledermatology", Journal of Medical Systems, Vol. 28, No. 5, October 2004

[17] C. Posse, "Projection Pursuit exploratory data analysis", Computational Statistics \& data analysis, Vol. 10, No 6, pp. 669-687, 1995.

[18] C. Posse, "Tools for two dimensional exploratory projection pursuit", $J$. Comput. Graph. Statist., Vol. 4, pp. 83-100, 1995.

[19] Taxt T., Hjort L., Eikvik L. : Statistical Classification using a Linear Mixture of two Multi-normal Probability Densities. Pattern Recognition Letters, Vol. 12, pp. 731-737, 1991. 